\title{
Significance of the centrally expressed TRP channel painless in Drosophila courtship memory
}

\author{
Takaomi Sakai, ${ }^{1}$ Shoma Sato, ${ }^{1}$ Hiroshi Ishimoto, ${ }^{2,4}$ and Toshihiro Kitamoto ${ }^{2,3,5}$ \\ ${ }^{1}$ Department of Biological Sciences, Tokyo Metropolitan University, Hachi-oji 192-0397, Tokyo, Japan; ${ }^{2}$ Department of Anesthesia, \\ University of lowa, lowa City, lowa 52242, USA; ${ }^{3}$ Interdisciplinary Graduate Programs in Genetics and Neuroscience, University of \\ lowa, lowa City, lowa 52242, USA
}

\begin{abstract}
Considerable evidence has demonstrated that transient receptor potential (TRP) channels play vital roles in sensory neurons, mediating responses to various environmental stimuli. In contrast, relatively little is known about how TRP channels exert their effects in the central nervous system to control complex behaviors. This is also true for the Drosophila TRP channel encoded by painless ( pain). The Pain TRP channel is expressed in a subset of sensory neurons and involved in behavioral responses to thermal, chemical, and mechanical stimuli. Its physiological roles in brain neurons, however, remain largely elusive. Using multiple mutant alleles and tranformants for pain, here we demonstrate that the brain-expressed Pain TRP channel is required for long-term memory (LTM), but not for short-lasting memory, induced by courtship conditioning in adult males. The courtship LTM phenotype in pain mutants was rescued by expressing wild-type pain temporarily, prior to conditioning, in adult flies. In addition, targeted expression of painRNAi in either the mushroom bodies (MBs) or insulin-producing cells (IPCs) resulted in defective courtship LTM. These results indicate that the Pain TRP channels in the $\mathrm{MBs}$ and IPCs control neuronal plasticity that is required for the formation of a certain type of long-lasting associative memory in Drosophila.
\end{abstract}

The transient receptor potential (TRP) channel superfamily is composed of a large group of six-membrane, cation-permeable ion channels that display diverse modes of activation. They are conserved throughout animal phylogeny and have a wide variety of biological functions in different tissues and cell types (Moran et al. 2004; Ramsey et al. 2006; Minke 2010). In the peripheral nervous system (PNS), TRP channels play critical roles in the transduction of various sensory stimuli (Clapham 2003; Moran et al. 2004). Although several members of the TRP channel family are expressed in the central nervous system (CNS) and are implicated in behavioral and neural plasticity (Zhou et al. 2008; Riccio et al. 2009), the physiological and behavioral roles of TRP channels in the CNS are still largely unexplored.

The Drosophila TRP channel gene painless (pain) is expressed in both the larval and adult nervous system, and is involved in the sensing of noxious stimuli (Tracey et al. 2003; Xu et al. 2006; Sokabe et al. 2008), behavioral responses to wasabi (Al-Anzi et al. 2006), larval social behavior (Xu et al. 2008), female sexual behavior (Sakai et al. 2009), negative geotaxis (Sun et al. 2009), and responses to mechanical stress (Sénatore et al. 2010). An enhancer-trap allele, pain ${ }^{G A L 4}$, was shown to drive expression of a GFP reporter gene in the larval PNS, in a pattern consistent with expression of the endogenous pain mRNA (Tracey et al. 2003). In addition, pain ${ }^{G A L 4}$ drives the reporter expression in the adult brain, including the mushroom body $(\mathrm{MB})$, a brain region important for various types of learning and memory (Al-Anzi et al. 2006; Xu et al. 2006). This suggested that the pain gene may play a role in the physiological process required for Drosophila memory.

\footnotetext{
4Present address: Laboratory for Cell Asymmetry, RIKEN Center for Developmental Biology, Kobe 650-0047, Hyogo, Japan

Article is online at http://www.learnmem.org/cgi/doi/10.1101//m.029041.112.
}

In order to examine the intriguing possibility that pain expressed in the brain is involved in processing memory, we used an ethologically relevant, associative learning paradigm in Drosophila known as courtship conditioning. In this paradigm, male flies having courted unreceptive, nonvirgin females subsequently suppress their courtship behavior, even toward receptive virgin females (Siegel and Hall 1979; Hall 1994). Courtship memory (as judged by a reduction in courtship activities of males that have been aversively "trained" with nonvirgin females) can be retained for different lengths of time depending on the training condition (McBride et al. 1999; Mehren et al. 2004; Sakai et al. 2004; Ishimoto et al. 2009). One-hour conditioning creates memory detectable for several hours (McBride et al. 1999), whereas 7-h conditioning results in long-term memory (LTM) lasting at least 5 d under our experimental conditions (Sakai et al. 2004; Ishimoto et al. 2009).

In this study, we utilized multiple mutant alleles for Drosophila pain and investigated the function of Pain TRP channels in processing memory induced by courtship conditioning. Our results demonstrate that normal courtship LTM, but not short-lasting memory, requires Pain in the adult brain. We further reveal that the Pain TRP channels expressed in the MBs and insulin-producing cells (IPCs) play a critical role in the formation of courtship LTM.

\section{Results}

pain mutant males are defective for long-term courtship memory

According to our previously published experimental protocol (Sakai et al. 2004; Ishimoto et al. 2009), courtship memory in wild-type males $5 \mathrm{~d}$ after 7 -h conditioning is reliably detected as a significant reduction in the courtship index (CI) of conditioned males compared to that of naive males. The CI is defined as the 
percentage of time spent in male courtship behaviors during the observation period. In this report, we refer to the memory detected $5 \mathrm{~d}$ after conditioning as "long-term courtship memory" or LTM.

Four mutant alleles of pain were tested for the effects of pain mutations on LTM. pain ${ }^{1}$, pain ${ }^{2}$, and pain $^{3}$ [a.k.a. EP(2)2451, $\mathrm{EP}(2) 2621$, and EP(2)2251] have been shown to carry an EP transposable element (Rørth et al. 1998) in either the putative 5'-flanking region or the noncoding first exon of the pain gene (Fig. 1A; Tracey et al. 2003). The pain ${ }^{G A L 4}$ allele was generated by Tracey et al. (2003) from pain ${ }^{1}$ by P-element replacement, and has an insertion of the GAL4 enhancer-trap element at the position of the EP element in pain ${ }^{1}$ (Fig. 1A). pain ${ }^{1}$ pain $^{3}$, and pain ${ }^{\text {GAL4 }}$ on the Canton-S genetic background are homozygous viable and both homozygotes and heterozygotes were tested for their courtship LTM. The majority of $\operatorname{pain}^{2}$ homozygotes with the same genetic background die during development. Because rare pain ${ }^{2}$ homozygous escapers die within several days of eclosion, we used only

A
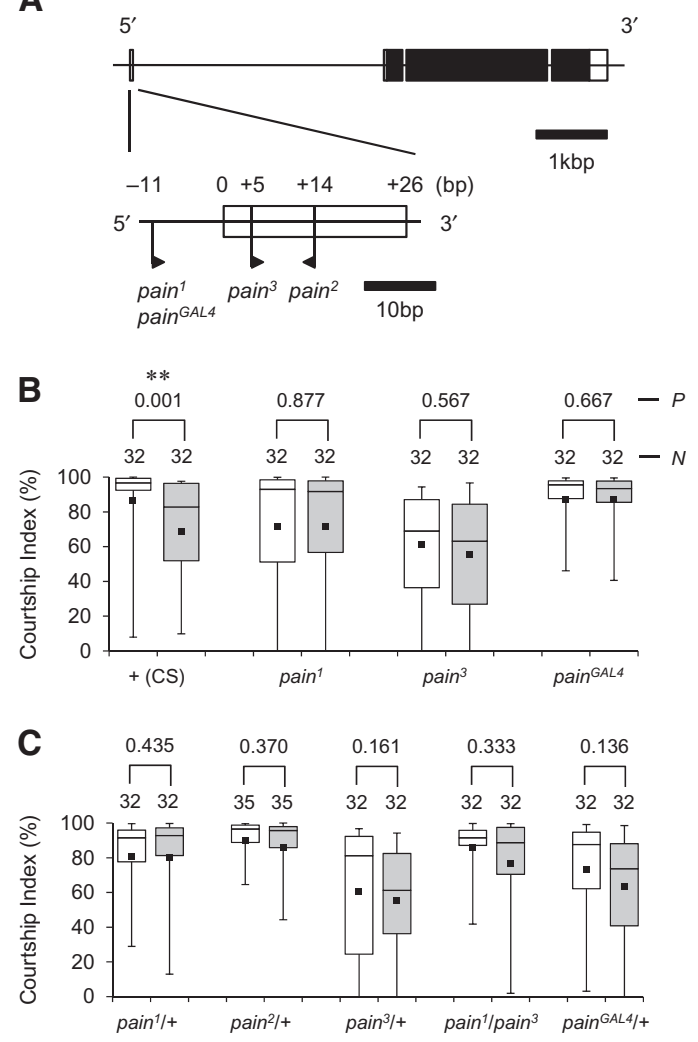

Figure 1. pain mutants are defective in courtship LTM. (A) Genomic structure of the pain gene. Boxes represent exons ((black) coding region, (white) noncoding region). P-element insertion sites of four pain mutants are indicated by flags. The orientation of the flag corresponds to the direction of transcription from the GAL4 binding site in the inserted EP element. Information on the genomic structure of the pain locus and the positions of transposon insertions was obtained from FlyBase (http: //flybase.org/reports/FBgn0060296.html). $(B, C) \mathrm{Cl}$ as an indicator of courtship memory. In each box plot, the box encompasses the interquartile range, a line is drawn at the median, and the vertical bars extend to the 5th and 95th percentiles. Each square within a box represents the mean. (White box) naive male, (gray box) conditioned male. $(P)$ probability, $(N)$ sample size, $\left({ }^{* *}\right) P<0.01$. (B) 5-d memory after 7-h conditioning, in wild-type flies and pain ${ }^{7}$, pain $^{3}$, and pain ${ }^{\text {GAL }}$ homozygotes. (C) 5-d memory after 7-h conditioning, in wild-type, $\operatorname{pain}^{1} /+$, $\operatorname{pain}^{2} /+$, $\operatorname{pain}^{3} /+$, $\operatorname{pain}^{1} /$ pain $^{3}$, and pain ${ }^{C A L 4} /+$ flies.
A

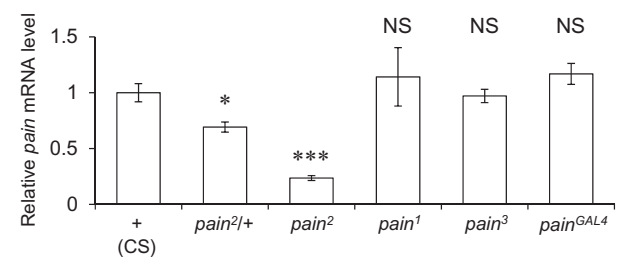

B

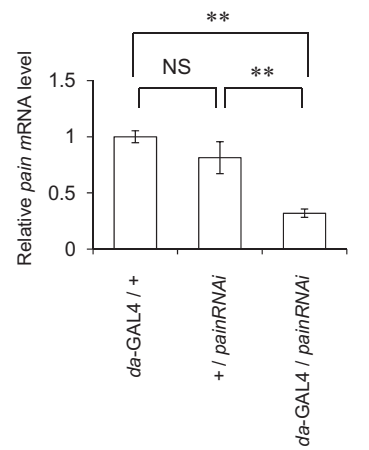

Figure 2. Real-time quantitative RT-PCR analysis of pain mRNA expression levels. (A) Wild-type (CS), pain ${ }^{2} /+$, pain $^{2}$, pain $^{1}$, pain $^{3}$, and pain ${ }^{\text {GAL4 }}$ males were used. Mean \pm SEM values were calculated for data resulting from 4-10 independent assays. Student's $t$-test was used to compare relative pain mRNA levels between wild type (CS) and each pain mutant. $\left(^{*}\right)$ $P<0.05$, (***) $P<0.001$, (NS) not significant. (B) da-GAL4/+, UASpainRNAi/+, and da-GAL4/UAS-painRNAi males were used. Mean \pm $S E M$ values were calculated for quadruplicated data. For multiple comparisons of relative pain mRNA levels among genotypes, one-way ANOVA with post-hoc Tukey's HSD test was used. ${ }^{(*)} P<0.01$, (NS) not significant, (painRNAi) UAS-painRNAi.

$\operatorname{pain}^{2} /+$ males to examine the effect of the $\operatorname{pain}^{2}$ mutation on courtship LTM.

Unlike wild-type males, mutant males homozygous for pain $^{1}$, pain ${ }^{3}$, or pain ${ }^{\text {GAL4 }}$ did not show an experience-dependent reduction in courtship activity $5 \mathrm{~d}$ after 7 -h conditioning, demonstrating that they are defective for courtship LTM (Fig. 1B). Males heterozygous for pain mutations (pain ${ }^{1}$, pain $^{2}$, pain $^{3}$, and pain $^{\text {GAL4 }}$ ) also displayed defective courtship LTM (Fig. 1C). This result was unexpected in light of the effect of pain mutations on nociception, for which pain ${ }^{1}$ and pain ${ }^{3}$ are recessive and pain ${ }^{2}$ is semidominant (Tracey et al. 2003). Our observations suggest that the courtship LTM is more susceptible to a reduction in Pain activity than nociceptive behavior is.

Real-time quantitative reverse transcription PCR (qRT-PCR) analysis revealed that levels of pain mRNA expression in pain ${ }^{2} /+$ and pain $^{2}$ are significantly lower than those in the wild-type (CS) flies. In contrast, pain ${ }^{1}$, pain $^{3}$, and pain ${ }^{\text {GAL4 }}$ did not significantly affect the pain mRNA expression level (Fig. 2A). Previous Western blots analysis showed that pain ${ }^{1}$ and pain $^{2}$ alleles produce Pain proteins with a molecular weight higher than that of the wild type, and that Pain protein expression in pain $^{2}$ is significantly reduced compared to those in the wild type and pain ${ }^{1}$ (Tracey et al. 2003). These data, combined with the result of our PCR analysis, suggest that the defective courtship LTM phenotype in pain mutants, particularly pain ${ }^{2} /+$, is caused by reduced amounts of functional Pain protein. There is also the possibility that some pain mutant alleles may produce mutant Pain proteins with aberrant activity, interfering with the wild-type pain function. The presence of such Pain proteins with dominant-negative properties could be the cause of defective LTM in flies heterozygous for pain mutations (Fig. 1C). 

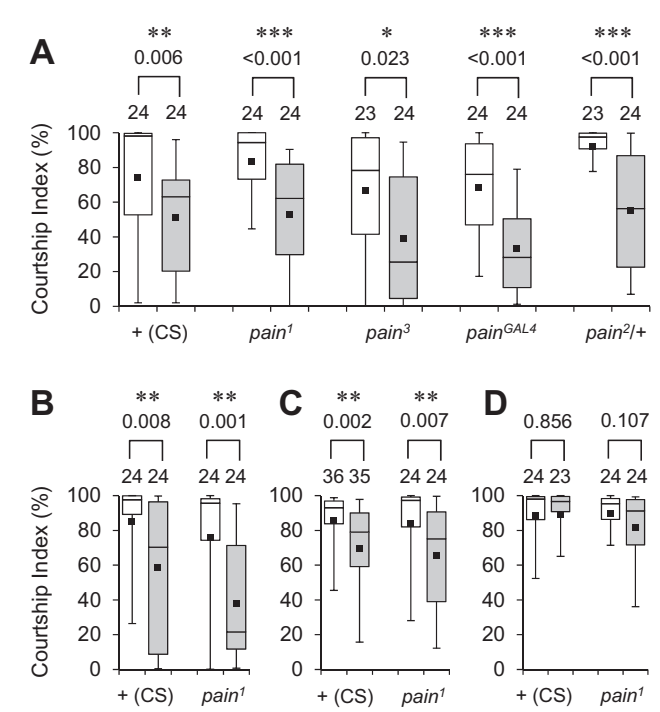

Figure 3. pain mutants are not defective in short-lasting courtship memory. (A) 30-min memory after 1-h conditioning in wild-type flies, pain $^{7}$, pain ${ }^{2}$, pain ${ }^{3}$, and pain ${ }^{\text {GAL4 }}$ homozygotes, and pain $^{2} /+$ heterozygotes. $\left(^{*}\right) P<0.05,\left(^{* *}\right) P<0.01,\left(^{* * *}\right) P<0.001$. (B) 1-h memory after 1 -h conditioning in wild-type flies and pain ${ }^{1}$ homozygotes. $(* *) P<$ 0.01 . (C) 8-h memory after 1 -h conditioning in wild-type flies and pain ${ }^{1}$ homozygotes. $\left({ }^{* *}\right) P<0.01$. (D) 24 -h memory after 1 -h conditioning in wild-type flies and pain ${ }^{1}$ homozygotes. (White box) naive male, (gray box) conditioned male.

\section{pain mutant males are not defective for short-lasting courtship memory}

To examine whether mutations in pain affect courtship memory in general, we measured short-lasting memory induced by 1-h conditioning. Like wild-type males, pain $^{1}$, pain ${ }^{3}$, and pain ${ }^{G A L 4}$ homozygotes and pain $^{2} /+$ males exhibited memory 30 min after 1-h conditioning (Fig. 3A). The quality of the short-lasting memory seems to be similar in wild-type and pain mutant males, because both in the presence and absence of a pain mutation, the memory was retained for $1 \mathrm{~h}$ and $8 \mathrm{~h}$ after 1-h courtship conditioning, and disappeared $24 \mathrm{~h}$ later (Fig. 3B-D). These results indicated that short-lasting memory induced by 1 -h conditioning is comparable in wild-type and pain mutant males, and that pain does not play a significant role in short-lasting courtship memory. Thus, the effects of pain on courtship memory appear to be selective for a long-lasting form.

\section{The LTM phenotype in pain mutants is rescued by expression of the wild-type pain gene}

Next, we examined courtship LTM in transheterozygous combinations of different pain mutant alleles. Males transheterozygous for pain $^{1}$ and pain $^{3}\left(\right.$ pain $^{1} /$ pain $^{3}$ ) failed to show courtship LTM (Fig. 1C), as expected from their dominant phenotype in LTM. In contrast, LTM was readily detected in particular transheterozygous combinations of the pain mutant alleles, namely pain ${ }^{G A L 4}$ / pain $^{1}$ and pain ${ }^{G A L 4} /$ pain $^{3}$ (Fig. 4A). In these flies, GAL4 expression from the pain ${ }^{G A L 4}$ allele likely resulted in the transcriptional initiation of pain from the GAL4 binding site in the EP element inserted in pain ${ }^{1}$ and $p^{2} a^{3}$ alleles. The GAL4/UAS-induced pain transcripts in pain ${ }^{1}$ and pain $^{3}$ are expected to produce the wildtype Pain protein, because they do not contain an upstream in-frame ATG (Tracey et al. 2003). This hypothesis is supported by the observation that $p a i n^{G A L 4} / p^{2 i n^{2}}$ flies were still defective for LTM (Fig. 4A); unlike pain ${ }^{\text {GAL4 }} /$ pain $^{1}$ and pain $^{\text {GAL4 }} /$ pain $^{3}$, pain $^{\text {GAL4 }} /$ pain $^{2}$ is not expected to produce wild-type Pain protein because the EP transposable element in $\mathrm{pain}^{2}$ is oriented in the opposite direction to that of the endogenous pain transcripts (Tracey et al. 2003). LTM was detected when a transgene encompassing the entire pain gene was introduced in pain ${ }^{\text {GAL4 }} /+$ and pain $^{2} /+$ heterozygotes (Fig. 4A), indicating that the defect in pain mutants can be overcome by expressing the functional wild-type pain gene at appropriate levels.

\section{Functional pain TRP channels are required in adult males for normal courtship LTM}

Although pain mutants have no obvious abnormality in overall adult brain morphology (Al-Anzi et al. 2006; Xu et al. 2006), it is possible that the observed LTM defect is caused by impaired development due to mutations in pain. To determine if pain expression in adulthood is critical for normal courtship LTM, we used a heatshock GAL4 (hs-GAL4) driver and examined the effects of conditional pain expression on the LTM phenotype in pain mutants (Fig. 4B). As expected from the dominant phenotype of pain ${ }^{1}$, in the absence of heat-shock, male flies transheterozygous for pain ${ }^{1}$ and $h s$-GAL4 ( pain $^{1} / h s$-GAL4) were defective in courtship LTM (Fig. 4C, HS-). In contrast, courtship LTM was observed when pain expression was induced by a 40 -min heat-shock at $37^{\circ} \mathrm{C}, 3 \mathrm{~h}$ before 7-h conditioning [Fig. 4C, HS+(1)]. pain $^{1} /+$ males without the $h s$-GAL4 transgene did not show courtship LTM after heatshock treatment (Fig. 4D). These results indicate that pain plays a role in the ongoing physiological process that is important for
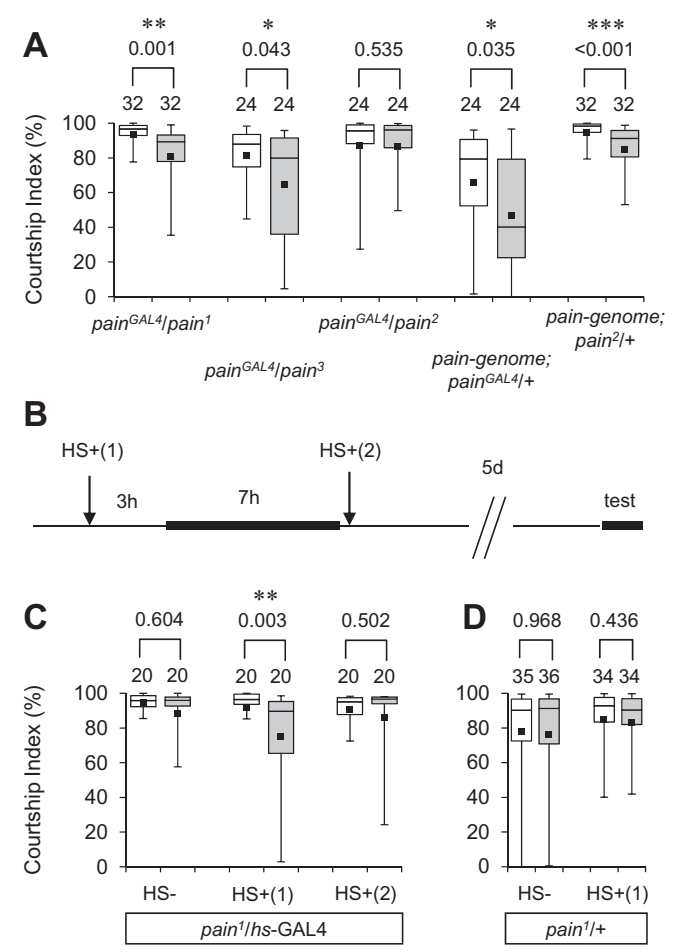

Figure 4. The LTM phenotype in pain mutants is rescued by the endogenous or transgenic pain gene. $(A)$ 5-d memory after 7-h conditioning in pain ${ }^{\text {GAL }} /$ pain $^{1}$, pain ${ }^{\text {GAL }} /$ pain $^{3}$, pain ${ }^{\text {GAL }} /$ pain $^{2}$, paingenome; pain ${ }^{C A L 4} /+$ and pain-genome; pain $^{2} /+$ flies. $(*) P<0.05,(* *)$ $P<0.01$, (***) $P<0.001$. (B) Experimental paradigms for heat-shock treatments. (HS-) non-heat-shocked males, $(\mathrm{HS}+(1))$ males heatshocked before conditioning, (HS+(2)) males heat-shocked after conditioning. (C) 5-d memory after 7-h conditioning in flies transheterozygous for pain ${ }^{1}$ and hs-GAL4 ( pain $^{1} /$ hs-GAL4) with or without a 40-min heatshock before $(\mathrm{HS}+(1))$ or after $(\mathrm{HS}+(2))$ conditioning. $(* *) P<0.01$. (D) 5-d memory after 7 -h conditioning in pain $^{1} /+$ males with or without a 40-min heat-shock. 
courtship LTM, and that reduction of pain activity during development does not have a significant effect on this behavioral plasticity. Moreover, induction of pain after 7-h conditioning failed to restore the ability of pain ${ }^{1}$ heterozygotes to show courtship LTM [Fig. 4C, HS+(2)], suggesting that pain is involved in the formation, rather than storage or retrieval, of courtship LTM.

pain transcripts are widely expressed in the adult CNS pain $^{\text {GAL4 }} /$ pain $^{1}$ and pain ${ }^{\text {GAL }} /$ pain $^{3}$ showed courtship LTM (Fig. $4 \mathrm{~A}$ ), indicating that pain expression in pain $^{G A L 4}$-positive neurons is important for normal courtship LTM. pain was suggested to be expressed in the adult CNS based on the fact that a pain GAL4 enhancer-trap allele ( pain $^{\text {GAL4 }}$ ) directs GFP reporter gene expression to the adult brain, with the most prominent signal appearing in the MBs (Tracey et al. 2003; Al-Anzi et al. 2006). We reexamined GFP reporter expression in the adult brain in pain ${ }^{\text {GAL4 }}$, UAS-GFP males (Fig. 5A,B) and confirmed that pain $^{\text {GAL4 }}$ drives GFP expression in various brain regions, including the MBs (Fig. 5A. arrows), the ellipsoid body (EB) of the central complex (CX) (Fig. 5B, triangle), and the pars intercerebralis (Fig. 5A, asterisk).

The pattern of pain ${ }^{\text {GAL4 }}$-driven reporter gene expression strongly suggests endogenous expression of pain in the adult brain. To further obtain evidence for pain expression in the central neurons, we carried out in situ hybridization analysis and directly examined the distribution of pain transcripts throughout the adult CNS. An antisense probe for pain revealed widespread expression in the neuronal cell body regions of the adult brain, albeit accurate identification of the pain transcript-positive cells was not obtained. No signal was observed when a sense probe was used under the same conditions (Fig. 5C). This result is consistent with the microarray data available from the Drosophila gene expression atlas (FlyAtlas) website (http://www.flyatlas. org/), which indicates that pain transcripts are present at relatively higher levels in the adult brain and thoracicoabdominal ganglion compared to other tissues (Chintapalli et al. 2007).

\section{Knockdown of pain in a subset of neurons mimics the pain mutant phenotype}

To further confirm the functional significance of the centrally expressed Pain TRP channels in courtship LTM, we employed the UAS-painRNAi transgene in combination with GAL4 drivers that direct expression of UAS-effector genes in subsets of brain neurons. The effectiveness of painRNAi was first confirmed by qRTPCR analysis, demonstrating that ubiquitous expression of painRNAi using the da-Gal4 driver results in an $\sim 70 \%$ reduction of pain expression relative to that in control flies (Fig. 2B). Because pain ${ }^{\text {GAL4 }}$ positive neurons include those in the
MBs, EB, and pars intercerebralis (Fig. 5A,B), the corresponding GAL4 lines were used to suppress pain expression in a neuronal subset-specific manner (Fig. 5D-J).

When painRNAi expression was directed to the MB, a brain structure intimately involved in learning and memory (Heisenberg 2003), using OK107, c772, 30Y, and MB247 (Fig. 5D-G) the resultant flies consistently displayed defects in courtship LTM (Fig. 6A). All control males (+/UAS-painRNAi, OK107/+, c772/+, 30Y/+, and MB247/+) exhibited LTM (Fig. 6B). Similar results were also obtained using a different UAS-painRNAi line (data not shown). These findings with UAS-painRNAi lines strongly suggest that Pain TRP channels in the MBs play a critical role in courtship LTM.

The CX is an integration center that receives input from various parts of the insect brain. It has been shown that neurons in the $\mathrm{CX}$ are required for visual memory (Pan et al. 2009). In contrast to the results with "MBs-GAL4 lines," males expressing painRNAi
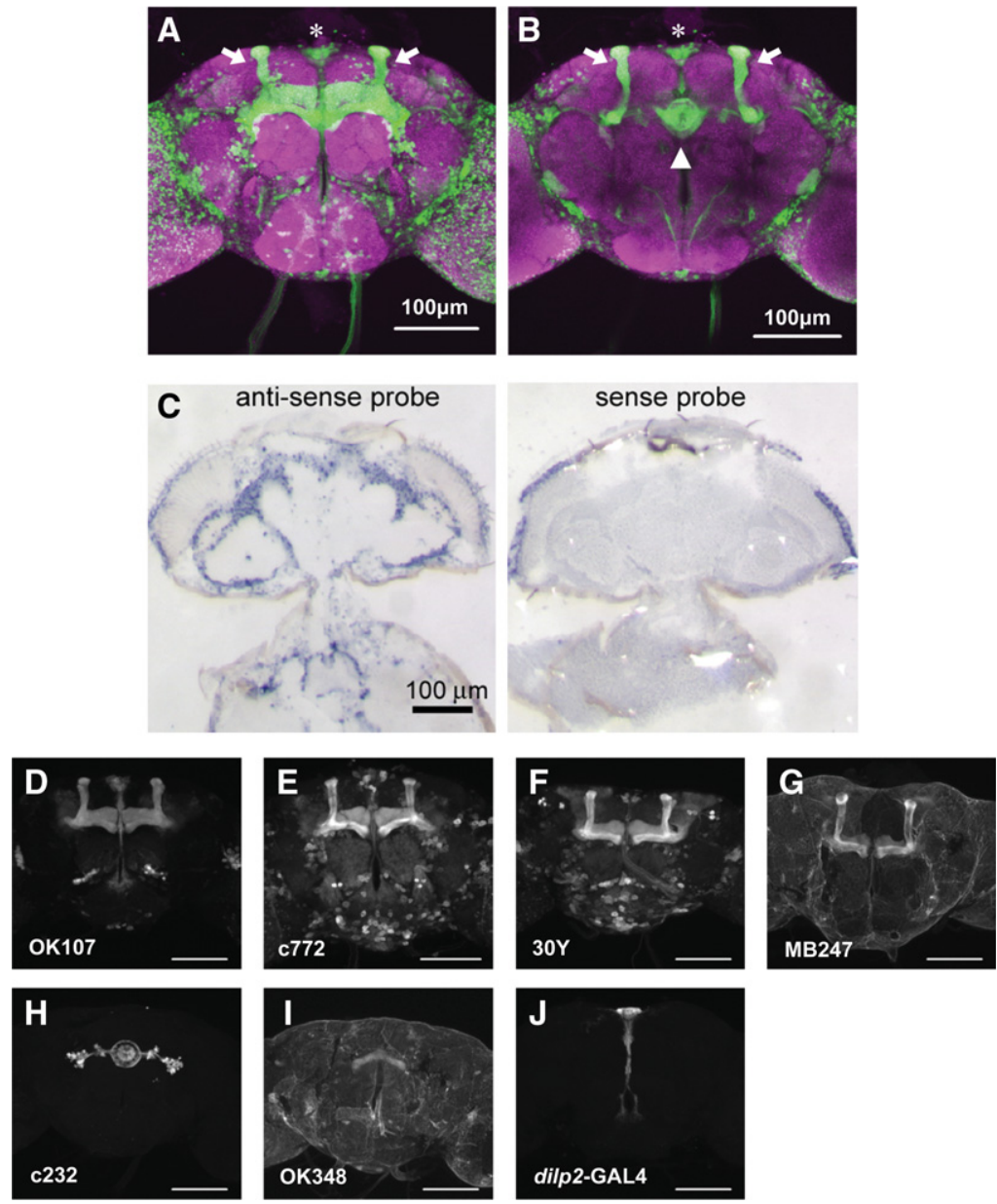

Figure 5. The widespread pain expression in the adult brain is deduced by pain ${ }^{G A L 4}$ and in situ hybridization analysis. $(A)$ A stacked confocal image (frontal view) and $(B)$ a section image (at the level of the $\mathrm{EB}$ ) of the adult brain. Homozygous males for pain ${ }^{G A L 4}$, UAS-GFP were used. Green and magenta show GFP fluorescence and counterstaining with an nc82 antibody, respectively. (Arrows) the MBs, (asterisk) the PI, (triangle) the EB in the CX. (C) In situ hybridization analysis with a DIG-labeled antisense RNA probe reveals that pain transcripts are present in cortical regions of the adult brain. The sense probe does not produce any significant signal in the brain. (D-J) GAL4 expression patterns in the adult brain in seven GAL4 lines, as visualized by GFP reporter expression. GFP expression in $F_{1}$ males from crosses between GAL4 lines (OK107 [D], c772 [E], 30Y $[F]$, MB247 [G], c232 [H], OK348 [I], dilp2-GAL4 [J]), and UAS-mCD8::GFP. c772, 30Y, OK107, and MB247 are MBs-GAL4 lines. In c232, the GFP signal is detected specifically in the ellipsoid body of the CX. OK348 is a GAL4 driver for the fan-shaped body of the CX. In dilp2-GAL4, the GFP signal is specifically detected in IPCs. Scale bars show $100 \mu \mathrm{m}$. 
A
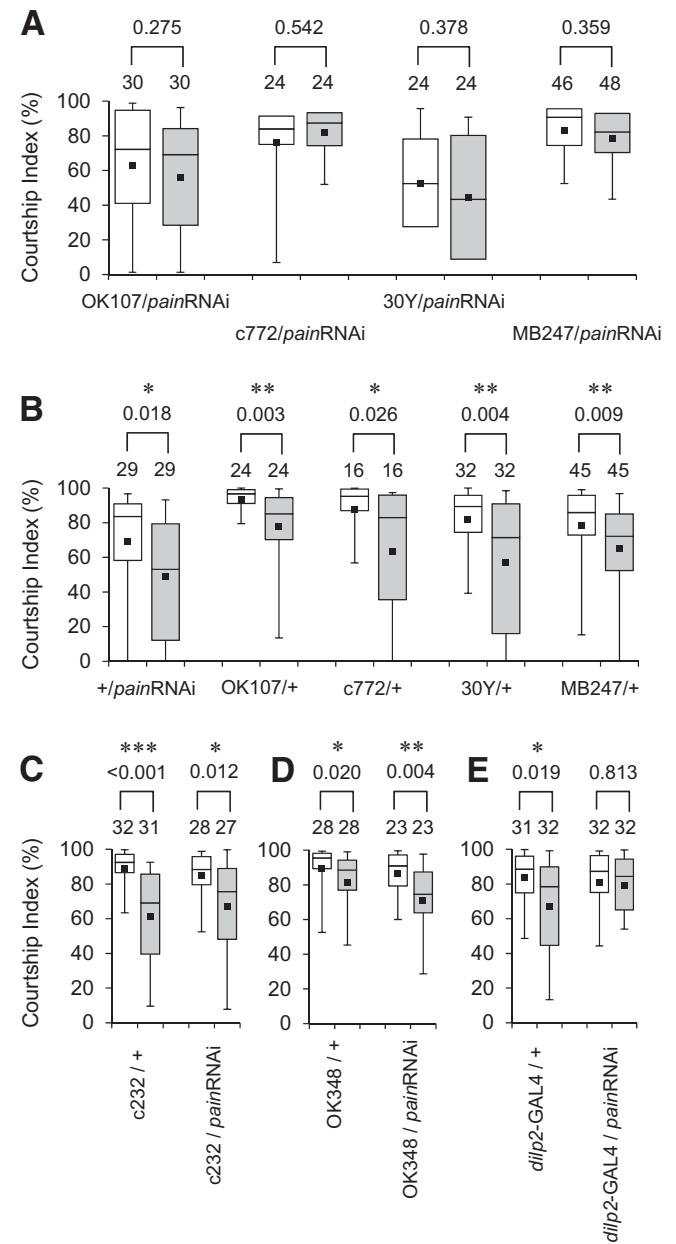

Figure 6. Neuronal subset-specific knockdown of pain mimics the pain mutant phenotype. A UAS-painRNAi line was crossed to GAL4 drivers that direct expression in various neuronal subpopulations. (A) 5-d memory after 7-h conditioning in $F_{1}$ male progeny from a cross between the UAS-painRNAi and the indicated MBs-GAL4 lines. (B) 5-d memory after 7-h conditioning in UAS and MBs-GAL4 control males. (C) 5-d memory after 7-h conditioning in c232/+ and c232/ UAS-painRNAi males. (D) 5-d memory after 7-h conditioning in OK348/+ and OK348/ UASpainRNAi males. (E) 5-d memory after 7-h conditioning in dilp2GAL4/+ and dilp2-GAL4/UAS-painRNAi males. (*) $\mathrm{P}<0.05$, (**) $\mathrm{P}<$ 0.01 , (***) $\mathrm{P}<0.001$. (White box) naive male, (gray box) conditioned male, (painRNAi) UAS-painRNAi.

specifically in the CX [c232 and OK348 lines (Fig. 5H,I)] showed courtship LTM (Fig. 6C,D). We also tested a Drosophila insulin-like peptide 2 (dilp2)-GAL4 line (Fig. 5J), directing gene expression specifically in IPCs, a cluster of neurons in the pars intercerebralis (Brogiolo et al. 2001). The dilp2-GAL4 transgene is composed of GAL4 cDNA fused to the regulatory region of the dilp2. Notably, expression of painRNAi in the brain IPCs resulted in the defective courtship LTM (Fig. 6E), indicating that Pain TRP channels in the IPCs are also required for courtship LTM.

\section{The LTM phenotype in pain mutants is rescued by Gal4-driven pain expression in the MBs or IPCs}

Next, we examined whether Gal4-driven pain expression in the MBs or IPCs has any effect on the defective LTM phenotype in pain $^{1} /+$ males. Interestingly, expression of pain in either the MBs by MB247 or the IPCs by dilp2-GAL4 rescued the LTM defect in pain $^{1} /+$ (Fig. 7). Thus, suppression of pain expression either in the MBs or in the IPCs of wild-type males resulted in defective courtship LTM, whereas Gal4-driven expression of pain either in the MBs or the IPCs led to restoration of LTM in pain $^{1} /+$ males. These results indicate that expression levels of Pain in these particular neuronal subsets are critical for the physiological process involved in courtship LTM.

\section{Discussion}

In this study we have identified a novel role of the brain-expressed Drosophila Pain TRP channel in the formation of courtship LTM. Because pain mutants do not show obvious abnormality in male courtship and they have the ability to acquire, store, and retrieve short-lasting courtship memory, their deficiency in LTM is likely due to a rather specific defect in the consolidation of labile memory into a long-lasting form of memory. What is the possible physiological mechanism by which Pain controls the formation of courtship LTM? A cell culture-based study demonstrated that Pain is a $\mathrm{Ca}^{2+}$-permeable channel and its activation is controlled by both extracellular and intracellular $\mathrm{Ca}^{2+}$ (Sokabe et al. 2008). Therefore, $\mathrm{Ca}^{2+}$ is one of the potential signals involved in the Pain-mediated formation of LTM. cAMP responsive element binding protein (CREB) is a transcriptional regulator that plays an essential role in the formation of LTM in both vertebrates and invertebrates (Kandel 2001). We previously demonstrated that induction of a repressor isoform of Drosophila CREB (dCREB2b) results in defective courtship LTM (Sakai et al. 2004), and that CREB-dependent transcription is enhanced by 7-h courtship conditioning (Ishimoto et al. 2009). These findings strongly indicate that courtship LTM requires training-dependent CREB activation. Because CREB can function as a $\mathrm{Ca}^{2+}$-inducible transcription factor (Lonze and Ginty 2002), it is possible that Pain contributes to LTM formation by modulating the calcium dynamics critical for CREB-mediated transcriptional regulation.

We have previously reported that pain mutations enhance sexual receptivity in Drosophila virgin females (Sakai et al. 2009). Interestingly, a similar phenotype in female sexual receptivity is observed when a repressor isoform of dCREB2 is conditionally expressed in adult females (Sakai and Kidokoro 2002), suggesting the possibility that dCREB2-mediated gene expression is under the control of the Pain TRP channels in the physiological processes responsible for normal female receptivity. Although female sexual receptivity and male courtship memory are likely controlled by distinct neural circuits, they may share, at least in part, the underlying regulatory mechanisms and be modified in an experiencedependent manner through both Pain and CREB.

Although pain mutants are defective in long-term courtship memory, they apparently show rather enhanced neural plasticity

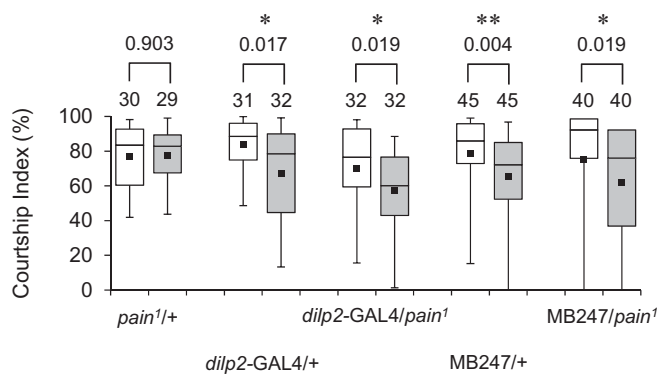

Figure 7. Neuronal subset-specific expression of pain rescues the pain mutant LTM phenotype. 5-d memory after 7-h conditioning in pain $^{1} /+$, dilp2-GAL4/+, dilp2-GAL4/pain $1, M B 247 /+$, and MB247/ pain $^{1}$ flies. (*) $P<0.05$, (**) $P<0.01$. (White box) naive male, (gray box) conditioned male. 
under certain circumstances. As mentioned above, female sexual receptivity is, in a sense, more plastic in pain mutants than in controls because the mutant females change their behavior from rejective to acceptive faster than controls do in response to a male's courtship (Sakai et al. 2009). In addition, a recent unpublished study has revealed that pain mutants exhibit an unusually rapid speed of habituation in the giant fiber (GF) pathway ( $\mathrm{Z}$ Wang and $\mathrm{CF} \mathrm{Wu}$, pers. comm.). The loci of this neuronal plasticity are known to reside in CNS circuits afferent to the GF pathway (Engel and $\mathrm{Wu}$ 1996). This rapid habituation phenotype of pain mutants indicates that certain CNS neurons in pain mutants are modified in an activity-dependent manner more easily than those in wild-type flies, and stop responding to the stimulus that originally evoked a response. We previously found that the duration of conditioning is crucial for courtship LTM: whereas 7-h conditioning leads to detectable LTM, 5-h conditioning is insufficient for inducing LTM in our experimental protocol (Sakai et al. 2004). During the 7-h conditioning period, certain brain neurons must be activated repeatedly to trigger stable changes in the relevant neuronal circuits, which is necessary for courtship LTM. A speculative hypothesis is that such critical brain neurons in pain mutants may not be activated sufficiently during 7-h conditioning, presumably because short-lasting modifications are easily induced in components of the corresponding circuits in pain mutants and disrupt their normal neuronal activities. As a result, although short-lasting memory is apparently normal in pain mutants, they are not able to form LTM using the standard experimental protocols because pain mutants are conditioned only insufficiently for LTM.

Courtship LTM was impaired when pain expression was knocked down by painRNAi in the MBs, a brain region important for courtship memory (Joiner and Griffith 1999; McBride et al. 1999). The GAL4 lines used to drive painRNAi in the MBs (OK107, c772, 30Y, and MB247) direct overlapping gene expression in the $\alpha / \beta$ and $\gamma$ neurons of the MBs (Aso et al. 2009), indicating that normal courtship LTM requires an intact level of Pain activity in these MB neurons. In addition to the MBs, we have discovered that Pain is required for courtship LTM in the neurosecretory IPCs-knockdown of pain expression in the neurosecretory IPCs resulted in defective courtship LTM. Furthermore, our analysis revealed that Gal4-driven expression of pain in the MBs or IPCs leads to restoration of courtship LTM in pain $^{1} /+$ males. This result was somewhat unexpected because RNAi experiments indicate that courtship LTM requires sufficient expression of functional Pain both in the MBs and IPCs. Although pain expression was directed only to the MBs (pain ${ }^{1} / \mathrm{MB} 247$ ) or IPCs (pain ${ }^{1} /$ dilp2- $^{1}$ GAL4), but not to both, courtship LTM was observed in $p^{2} i^{1} /+$ males. Apparently, overexpression of pain in the MBs compensates insufficient Pain activity in the IPCs. Similarly, pain overexpression in the IPCs compensates insufficient Pain activity in the MBs. Further studies are required for a better understanding the molecular and cellular underpinnings of the potential functional interactions between the MBs and IPCs in the context of Pain-mediated regulation of LTM.

This study revealed that functional Pain expression in the IPCs is required for courtship LTM. Because the IPCs are neurosecretory cells, this result raises the possibility that the Pain TRP channel contributes to the regulated secretion of hormones, such as insulin-like peptides, from the IPCs, and that hormones derived from the IPCs play an important role in consolidating memory to a long-lasting form. This is particularly interesting in light of several observations that insulin signaling is linked to experience-dependent behavioral modifications. In the rat, insulin-like growth factor II enhances memory retention and prevents forgetting (Chen et al. 2011), while in the nematode, mutations in the insulin-signaling pathway result in severe defects in salt che- motaxis learning (Tomioka et al. 2006). In Drosophila, insulin signaling plays important roles in regulating behaviors such as locomotor activity and the response to ethanol (Corl et al. 2005; Belgacem and Martin 2006). Furthermore, recently a systemic metabolic cue triggered by insulin signaling has been shown to influence food searches by modulating olfactory sensitivity (Root et al. 2011). These findings have demonstrated that the internal physiological states controlled by insulin play an important role in the regulation of behavioral plasticity. Therefore, it is of particular importance to determine how insulin signaling is regulated by Pain during courtship conditioning, and how it affects the functional properties of the brain neural circuits that are critical for normal courtship LTM, such as those involving the MBs.

\section{Materials and Methods}

\section{Fly stocks}

Wild-type Drosophila melanogaster Canton-S (CS), pain mutants (pain $^{1}$, pain $^{2}$, pain $^{3}$, and pain ${ }^{\text {GAL4 }}$ ), UAS-painRNAi, MBs-GAL4 lines (OK107, c772, 30Y, and MB247), c232, OK348, UAS$m C D 8:: G F P$, and dilp2-GAL4 were raised on a glucose-yeast-cornmeal medium at $25.0 \pm 0.5^{\circ} \mathrm{C}$ in a 12 -h light:12-h dark (LD) cycle. pain mutants, c772, 30Y, MB247, c232, OK348, and dilp2-GAL4 were outcrossed for at least six generations to white flies with the CS genetic background. Virgin males or females were collected without anesthesia within $6 \mathrm{~h}$ of eclosion, and were maintained in vials until used in the experiments.

\section{Courtship conditioning assay}

The courtship conditioning assay was performed as described previously (Sakai et al. 2004), with some modifications. Unreceptive, mated females were prepared as "trainers" a day before they were used for courtship conditioning by placing a 5- to 7-d old virgin CS female and a 5- to 7-d old virgin male in a courtship chamber made of transparent acrylic plastic $(15 \mathrm{~mm}$ in diameter $\times 3 \mathrm{~mm}$ in depth) for copulation. Conditioning for short-lasting memory assays involved pairing a 3 - to 4 -d-old virgin male with the trainer female for $1 \mathrm{~h}$ in a conditioning chamber $(15 \mathrm{~mm}$ in diameter $\times$ $5 \mathrm{~mm}$ in depth) containing food medium. In the case of conditioning for LTM assays, a male was paired with the trainer female for $7 \mathrm{~h}$. Conditioned males were kept in a glass test tube $(12 \mathrm{~mm}$ in diameter $\times 75 \mathrm{~mm}$ in depth) containing food medium until tested with a freeze-killed virgin female as a courtship target. Memory tests for the short-lasting and LTM assays were performed in the courtship chamber $30 \mathrm{~min}, 1 \mathrm{~h}, 8 \mathrm{~h}, 24 \mathrm{~h}$, and $5 \mathrm{~d}$ after conditioning. The CI was defined as the percentage of 10 minutes that was spent in courtship behaviors. Courtship memory was considered to be present when CI values of conditioned males were significantly $(P<0.05)$ lower than those of naive males. In most cases, CI was not distributed normally. Thus, we carried out the arcsine transformation of CI. When the transformed values showed the normal distribution and homoscedasticity was evident, they were analyzed by Student's $t$-test. When the transformed values did not show the normal distribution or homoscedasticity was not evident, we used a nonparametric test (Mann-Whitney $U$-test). Computer software (PASW Statistics 18) was used for statistical analysis.

\section{Real-time quantitative reverse transcription PCR}

Total RNA was isolated from approximately 50 male fly heads of each genotype using the RNeasy Mini Kit (QIAGEN). cDNA was synthesized by carrying out a reverse transcription reaction using the QuantiTect Reverse Transcription Kit (QIAGEN). Real-time quantitative PCR was carried out using SYBR Premix Ex Taq (Takara Bio Inc.) and a Chromo 4 Detector (MJ Research). Levels of pain mRNA were normalized by those of $r p 49$ mRNA. The average of normalized pain mRNA levels in control flies (CS in Fig. 2A and $d a-G A L 4 /+$ in Fig. 2B) was calculated for data resulting from 4-10 independent assays. The ratio between normalized pain mRNA levels in each experimental genotype and the averaged 
control value was calculated. The mean $( \pm$ SEM) of the ratio values was calculated for data resulting from 4-10 independent assays. The primer sequences used for real-time PCR were as follows: pain-forward, 5'-CACTCTCAACACCAGGTTGTC-3'; pain-reverse, 5'-AGGTTTCCTGGATCCCTAGAG-3'; rp49-forward, $\quad$ 5'-AAGA TCGTGAAGAAGCGCAC-3'; rp49-reverse, 5'-TGTGCACCAGGAA CTTCTTG $-3^{\prime}$.

\section{In situ hybridization}

In situ hybridization was performed, using an antisense digoxigenin (DIG)-labeled RNA probe prepared with the DIG RNA labeling kit (Roche Applied Science), on adult fly cryosections $(10 \mu \mathrm{m})$. The antisense RNA was prepared from the SP6 promoter of a pCRII clone containing the painless cDNA. A sense probe was transcribed from the T7 promoter and used as a negative control. The sectioned samples were fixed with $3.7 \%$ paraformaldehyde in PBS for $30 \mathrm{~min}$, and then washed three times for $15 \mathrm{~min}$ in PBS $/ 0.1 \%$ Triton X-100 (PBST). Hybridization was performed at $50^{\circ} \mathrm{C}$ in hybridization buffer $(1 \mathrm{mg} / \mathrm{mL}$ tRNA, $20 \mathrm{mM}$ TrisHCl, $4 \mathrm{mM}$ EDTA pH 8.0, $1 \times$ Denhard's, $300 \mathrm{mM} \mathrm{NaCl}, 50 \%$ formamide, and $5 \%$ dextran sulfate) for $16 \mathrm{~h}$. Following hybridization with the RNA probe, samples were washed with a wash buffer $(2 \times$ SSC and $50 \%$ formamide). After the buffer was replaced with PBST, a sample slide was blocked with PBST with 1\% normal goat serum for $1 \mathrm{~h}$. The anti-DIG AP Fab fragment antibody (Roche Applied Science) was added at a 1:2000 dilution and incubated overnight at $4^{\circ} \mathrm{C}$. Following incubation with the antibody, samples were washed with PBST (three times for $15 \mathrm{~min}$ ) and incubated in NBT/BCIP solution for color development.

\section{Immunohistochemistry and microscopy}

Adult brains were stained with an anti-Bruchpilot mouse monoclonal antibody (1:40) (The Developmental Studies Hybridoma Bank at the University of Iowa, nc82). Alexa Fluor 568 anti-mouse IgG (Invitrogen) was used as a secondary antibody (1:1000). Fluorescence was observed using a confocal microscope (Carl Zeiss LSM710). For confocal microscopy, Z sections were collected at $1-\mu \mathrm{m}$ intervals and processed to construct projections through an extended depth of focus.

\section{Acknowledgments}

This work was supported by a Grant-in-Aid for Scientific Research on Innovative Areas "Systems Molecular Ethology" (to T.S.), a Grant-in-Aid for Scientific Research (B) (to T.S.), and partly by National Institutes of Health grants R01MH062684 and MH085081 (to T.K.). We thank the late Seymour Benzer ${ }^{6}$ and W. Daniel Tracey Jr for all pain mutants, and the late Yoshiaki Kidokoro, ${ }^{6}$ Toshiro Aigaki, Junjiro Horiuchi, Minoru Saitoe, and Kohei Ueno for helpful discussion.

\section{References}

Al-Anzi B, Tracey WD, Benzer S. 2006. Response of Drosophila to wasabi is mediated by painless, the fly homolog of mammalian TRPA1/ANKTM1. Curr Biol 16: 1034-1040.

Aso Y, Grübel K, Busch S, Friedrich AB, Siwanowicz I, Tanimoto H. 2009. The mushroom body of adult Drosophila characterized by GAL4 drivers. J Neurogenet 23: 156-172.

Belgacem YH, Martin JR. 2006. Disruption of insulin pathways alters trehalose level and abolishes sexual dimorphism in locomotor activity in Drosophila. J Neurobiol 66: 19-32.

Brogiolo W, Stocker H, Ikeya T, Rintelen F, Fernandez R, Hafen E. 2001. An evolutionarily conserved function of the Drosophila insulin receptor and insulin-like peptides in growth control. Curr Biol 11: 213-221.

Chen DY, Stern SA, Garcia-Osta A, Saunier-Rebori B, Pollonini G, Bambah-Mukku D, Blitzer RD, Alberini CM. 2011. A critical role for IGF-II in memory consolidation and enhancement. Nature 469: 491-497.

\footnotetext{
${ }^{6}$ Deceased
}

Chintapalli VR, Wang J, Dow JA. 2007. Using FlyAtlas to identify better Drosophila melanogaster models of human disease. Nat Genet 39: $715-720$.

Clapham DE. 2003. TRP channels as cellular sensors. Nature 426: 517-524.

Corl AB, Rodan AR, Heberlein U. 2005. Insulin signaling in the nervous system regulates ethanol intoxication in Drosophila melanogaster. Nat Neurosci 8: 18-19.

Engel JE, Wu CF. 1996. Altered habituation of an identified escape circuit in Drosophila memory mutants. J Neurosci 16: 3486-3499.

Hall JC. 1994. The mating of a fly. Science 264: 1702-1714.

Heisenberg M. 2003. Mushroom body memoir: From maps to models. Nat Rev Neurosci 4: 266-275.

Ishimoto H, Sakai T, Kitamoto T. 2009. Ecdysone signaling regulates courtship long-term memory formation in adult Drosophila melanogaster. Proc Natl Acad Sci 106: 6381-6386.

Joiner MA, Griffith LC. 1999. Mapping of the anatomical circuit of CaM kinase-dependent courtship conditioning in Drosophila. Learn Mem 6: $177-192$.

Kandel ER. 2001. The molecular biology of memory storage: A dialogue between genes and synapses. Science 294: 1030-1038.

Lonze BE, Ginty DD. 2002. Function and regulation of CREB family transcription factors in the nervous system. Neuron 35: 605-623.

McBride SM, Giuliani G, Choi C, Krause P, Correale D, Watson K, Baker G, Siwicki KK. 1999. Mushroom body ablation impairs short-term memory and long-term memory of courtship conditioning in Drosophila melanogaster. Neuron 24: 967-977.

Mehren JE, Ejima A, Griffith LC. 2004. Unconventional sex: Fresh approaches to courtship learning. Curr Opin Neurobiol 14: 745-750.

Minke B. 2010. The history of the Drosophila TRP channel: The birth of a new channel superfamily. J Neurogenet 24: 216-233.

Moran MM, Xu H, Clapham DE. 2004. TRP ion channels in the nervous system. Curr Opin Neurobiol 14: 362-369.

Pan Y, Zhou Y, Guo C, Gong H, Liu L. 2009. Differential roles of the fanshaped body and the ellipsoid body in Drosophila visual pattern memory. Learn Mem 16: 289-295.

Ramsey IS, Delling M, Clapham DE. 2006. An introduction to TRP channels. Annu Rev Physiol 68: 619-647.

Riccio A, Li Y, Moon J, Kim KS, Smith KS, Rudolph U, Gapon S, Yao GL, Tsvetkov E, Rodig SJ, et al. 2009. Essential role for TRPC5 in amygdala function and fear-related behavior. Cell 137: 761-772.

Root CM, Ko KI, Jafari A, Wang JW. 2011. Presynaptic facilitation by neuropeptide signaling mediates odor-driven food search. Cell 145: $133-144$.

Rørth P, Szabo K, Bailey A, Laverty T, Rehm J, Rubin GM, Weigmann K, Milán M, Benes V, Ansorge W, et al. 1998. Systematic gain-of-function genetics in Drosophila. Development 125: 1049-1057.

Sakai T, Kidokoro Y. 2002. Overexpression of a CREB repressor isoform enhances the female sexual receptivity in Drosophila. Behav Genet 32: 413-422.

Sakai T, Tamura T, Kitamoto T, Kidokoro Y. 2004. A clock gene, period, plays a key role in long-term memory formation in Drosophila. Proc Natl Acad Sci 101: $16058-16063$.

Sakai T, Kasuya J, Kitamoto T, Aigaki T. 2009. The Drosophila TRPA channel, Painless, regulates sexual receptivity in virgin females. Genes Brain Behav 8: 546-557.

Sénatore S, Rami Reddy V, Sémériva M, Perrin L, Lalevée N. 2010. Response to mechanical stress is mediated by the TRPA channel Painless in the Drosophila heart. PLoS Genet 6: e1001088. doi: 10.1371/ journal.pgen.1001088.

Siegel RW, Hall JC. 1979. Conditioned responses in courtship behavior of normal and mutant Drosophila. Proc Natl Acad Sci 76: 3430-3434.

Sokabe T, Tsujiuchi S, Kadowaki T, Tominaga M. 2008. Drosophila painless is a $\mathrm{Ca}^{2+}$-requiring channel activated by noxious heat. J Neurosci $\mathbf{2 8}$ 9929-9938.

Sun Y, Liu L, Ben-Shahar Y, Jacobs JS, Eberl DF, Welsh MJ. 2009. TRPA channels distinguish gravity sensing from hearing in Johnston's organ. Proc Natl Acad Sci 106: 13606-13611.

Tomioka M, Adachi T, Suzuki H, Kunitomo H, Schafer WR, Iino Y. 2006. The insulin/PI 3-kinase pathway regulates salt chemotaxis learning in Caenorhabditis elegans. Neuron 51: 613-625.

Tracey WD, Wilson RI, Laurent G, Benzer S. 2003. painless, a Drosophila gene essential for nociception. Cell 113: 261-273.

Xu SY, Cang CL, Liu XF, Peng YQ, Ye YZ, Zhao ZQ, Guo AK. 2006. Thermal nociception in adult Drosophila: Behavioral characterization and the role of the painless gene. Genes Brain Behav 5: 602-613.

Xu J, Sornborger AT, Lee JK, Shen P. 2008. Drosophila TRPA channel modulates sugar-stimulated neural excitation, avoidance and social response. Nat Neurosci 11: 676-682.

Zhou J, Du W, Zhou K, Tai Y, Yao H, Jia Y, Ding Y, Wang Y. 2008. Critical role of TRPC6 channels in the formation of excitatory synapses. Nat Neurosci 11: $741-743$.

Received October 18, 2012; accepted in revised form November 5, 2012. 


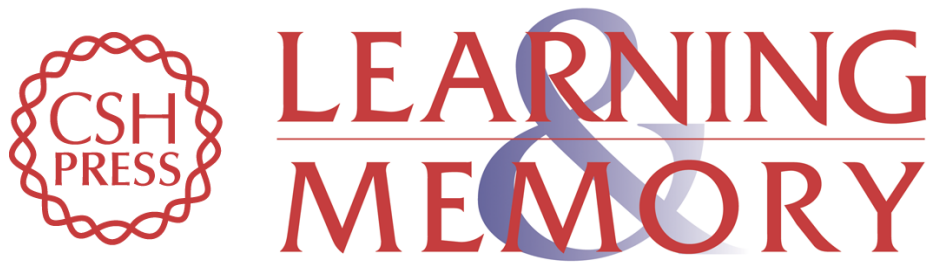

\section{Significance of the centrally expressed TRP channel painless in Drosophila courtship memory}

Takaomi Sakai, Shoma Sato, Hiroshi Ishimoto, et al.

Learn. Mem. 2013, 20:

Access the most recent version at doi:10.1101/lm.029041.112

References This article cites 36 articles, 11 of which can be accessed free at: http://learnmem.cshlp.org/content/20/1/34.full.html\#ref-list-1

License

Email Alerting Receive free email alerts when new articles cite this article - sign up in the box at the Service top right corner of the article or click here. 